\title{
Self-Adjointness, Symmetries, and Conservation Laws for a Class of Wave Equations Incorporating Dissipation
}

\author{
Yang Wang and Long Wei \\ Institute of Applied Mathematics and Engineering Computations, Hangzhou Dianzi University, Zhejiang 310018, China \\ Correspondence should be addressed to Long Wei; alongwei@gmail.com
}

Received 17 February 2013; Revised 30 April 2013; Accepted 13 May 2013

Academic Editor: Qian Guo

Copyright (C) 2013 Y. Wang and L. Wei. This is an open access article distributed under the Creative Commons Attribution License, which permits unrestricted use, distribution, and reproduction in any medium, provided the original work is properly cited.

In this work, we study the nonlinear self-adjointness and conservation laws for a class of wave equations with a dissipative source. We show that the equations are nonlinear self-adjoint. As a result, from the general theorem on conservation laws proved by Ibragimov and the symmetry generators, we find some conservation laws for this kind of equations.

\section{Introduction}

There have been abundant literatures that have contributed to the studies of Lie symmetry classification of various classes of $(1+1)$-dimensional nonlinear wave equations and their individual members. Probably, Barone et al. [1] were the first to study the nonlinear wave equation $u_{t t}=u_{x x}+F(u)$ by means of symmetry method. Motivated by a number of physical problems, Ames et al. [2] investigated group properties of quasilinear $u_{t t}=\left[f(u) u_{x}\right]_{x}$. Later, Torrisi and Valenti $[3,4]$, generalizing above equations, have investigated the symmetries of the following equation:

$$
u_{t t}=\left[f(u) u_{x}+g(x, u)\right]_{x} .
$$

Furthermore, classification results for the equation $u_{t t}+$ $K(u) u_{t}=\left[F(u) u_{x}\right]_{x}$ can be found in $[5,6]$. An expanded form of the latter equation

$$
u_{t t}+K(u) u_{t}=\left[F(u) u_{x}\right]_{x}+H(u) u_{x}
$$

was studied by Kingston and Sophocleous [7]. In the papers mentioned above, many interesting results including Lie point and nonlocal symmetries classification were systematically investigated.

In this paper, we consider a subclass of (2) with $K(u)=\alpha$ :

$$
u_{t t}+\alpha u_{t}-\left[f(u) u_{x}+g(u)\right]_{x}=0,
$$

which is also viewed as a special case of (1) with an additional dissipation, where $f(u)$ and $g(u)$ are arbitrary differentiable functions, $t$ is the time coordinate, $x$ is the one-space coordinate, and $\alpha$ is a nonzero constant. Here, we will focus on the nonlinear self-adjointness and conservation laws for (3).

For (3), we cannot find easily the variational structure so it is inconvenient to apply the Noether theorem to construct conservation laws straightforward for this equation. However, it is fortunate that Ibragimov recently proved a result on conservation laws [8], which does not require the existence of a Lagrangian. The Ibragimov theorem on conservation laws provides an elegant way to establish local conservation laws for the equations under consideration.

Since the seminal work of Ibragimov [8], more and more works are dedicated to studying the self-adjointness and conservation laws for some equations in mathematical physics and there are many new developments, including strict selfadjointness [9-11], quasi-self-adjointness [12-15], weak selfadjointness [16, 17], and nonlinear self-adjointness [18-21] and some results which have been communicated in the recent literature; for more references, see [21] and references therein.

For the sake of completeness, we briefly present the notations, definition of nonlinear self-adjointness, and Ibragimov's theorem on conservation laws. Let $x=\left(x_{1}, x_{2}, \ldots, x_{n}\right)$ be $n$ independent variables, $u=u(x)$ a dependent variable,

$$
X=\xi^{i} \frac{\partial}{\partial x_{i}}+\eta \frac{\partial}{\partial u}
$$


a symmetry of an equation

$$
\begin{gathered}
F\left(x, u_{(1)}, u_{(2)}, \ldots, u_{(s)}\right)=0 \\
F^{*}\left(x, u_{(1)}, v_{(1)}, \ldots, u_{(s)}, v_{(s)}\right):=\frac{\delta \mathscr{L}}{\delta u}=0
\end{gathered}
$$

the adjoint equation to (5), where $\mathscr{L}=v F$ is called formal Lagrangian, $v=v(x)$ is a new dependent variable, and

$$
\frac{\delta}{\delta u}=\frac{\partial}{\partial u}+\sum_{m=1}^{s}(-1)^{m} D_{i_{1}} \cdots D_{i_{m}} \frac{\partial}{\partial u_{i_{1} \cdots i_{m}}}
$$

denotes the Euler-Lagrange operator.

Now, let us state the definition of nonlinear self-adjointness for a equation.

Definition 1. Equation (5) is said to be nonlinearly self-adjoint if the equation obtained from the adjoint equation (6) by the substitution $v=\phi(x, u)$ with a certain function $\phi(x, u) \neq 0$ is identical with the original equation (5); in other words, the following equations hold:

$$
\left.F^{*}\right|_{v=\phi}=\lambda\left(x, u, u_{(1)}, \ldots\right) F
$$

for some differential function $\lambda=\lambda\left(x, u, u_{(1)}, \ldots\right)$.

Particularly, if (8) holds for a certain function $\phi$ such that $\phi_{u} \neq 0$ and $\phi_{x_{i}} \neq 0$ for some $x_{i},(5)$ is called weak self-adjoint; if (8) holds for a certain function $\phi$ such that $\phi=\phi(u) \neq u$ and $\phi^{\prime}(u) \neq 0$, then (5) is called quasi-self-adjoint; if (8) holds for $\phi=u$, then (5) is called (strictly) self-adjoint.

In the following, we recall the "new conservation theorem" given by Ibragimov in [8]. We will find conservation laws for (3) by this theorem.

Theorem 2. Any Lie point, Lie-Bäcklund, and nonlocal symmetry

$$
X=\xi^{i} \frac{\partial}{\partial x_{i}}+\eta \frac{\partial}{\partial u}
$$

of (5) provides a conservation law $D_{i}\left(C^{i}\right)=0$ for the system comprising (5) and its adjoint equation (6). The conserved vector is given by

$$
\begin{gathered}
C^{i}=\xi^{i} \mathscr{L}+W\left[\frac{\partial \mathscr{L}}{\partial u_{i}}-D_{j}\left(\frac{\partial \mathscr{L}}{\partial u_{i j}}\right)+D_{k} D_{j}\left(\frac{\partial \mathscr{L}}{\partial u_{i j k}}\right)\right. \\
\left.-D_{l} D_{k} D_{j}\left(\frac{\partial \mathscr{L}}{\partial u_{i j k l}}\right)+\cdots\right] \\
+D_{j}(W)\left[\frac{\partial \mathscr{L}}{\partial u_{i j}}-D_{k}\left(\frac{\partial \mathscr{L}}{\partial u_{i j k}}\right)+\cdots\right] \\
+D_{k} D_{j}(W)\left[\frac{\partial \mathscr{L}}{\partial u_{i j k}}-D_{l}\left(\frac{\partial \mathscr{L}}{\partial u_{i j k l}}\right)+\cdots\right]+\cdots,
\end{gathered}
$$

where $W=\eta-\xi^{j} u_{j}$ is the Lie characteristic function and $\mathscr{L}=$ $v F$ is the formal Lagrangian.
The paper is organized as the follows. In Section 2, we discuss the nonlinear self-adjointness of (3). In Section 3, we establish conservation laws for some particular cases of (3) using Theorem 2.

\section{Nonlinear Self-Adjointness of (3)}

In this section, we determine the nonlinearly self-adjoint subclasses of (3). Let

$$
F=u_{t t}+\alpha u_{t}-f_{u}(u) u_{x}^{2}-f(u) u_{x x}-g_{u}(u) u_{x} ;
$$

then we have the following formal Lagrangian for (3):

$$
\mathscr{L}=v\left(u_{t t}+\alpha u_{t}-f_{u} u_{x}^{2}-f u_{x x}-g_{u} u_{x}\right) .
$$

Computing the variational derivative of this formal Lagrangian

$$
\frac{\delta \mathscr{L}}{\delta u}=\frac{\partial \mathscr{L}}{\partial u}-D_{t} \frac{\partial \mathscr{L}}{\partial u_{t}}-D_{x} \frac{\partial \mathscr{L}}{\partial u_{x}}+D_{t}^{2} \frac{\partial \mathscr{L}}{\partial u_{t t}}+D_{x}^{2} \frac{\partial \mathscr{L}}{\partial u_{x x}},
$$

we obtain the adjoint equation of (3):

$$
F^{*}=-\alpha v_{t}+v_{t t}-f v_{x x}+g_{u} v_{x}=0 .
$$

Assume that $\left.F^{*}\right|_{v=h(t, x, u)}=\lambda F$, for a certain function $\lambda$, where $F$ is given by (11); then we have

$$
\begin{aligned}
h_{u} u_{t t} & +h_{u u} u_{t}^{2}+\left(2 h_{t u}-\alpha h_{u}\right) u_{t}-f h_{u} u_{x x}-f h_{u u} u_{x}^{2} \\
& +\left(g_{u} h_{u}-2 f h_{x u}\right) u_{x}+h_{t t}-\alpha u_{t}-f h_{x x}+g_{u} h_{x} \\
= & \lambda\left(u_{t t}+\alpha u_{t}-f_{u} u_{x}^{2}-f u_{x x}-g_{u} u_{x}\right) .
\end{aligned}
$$

The comparison of the coefficients of $u_{t t}, u_{t}^{2}, u_{x}^{2}, u_{t}$, and $u_{x}$ in both sides of (15) yields

$$
\begin{gathered}
\lambda=h_{u}, \\
h_{u u}=0, \\
\lambda f_{u}=f h_{u u}, \\
\lambda \alpha=2 h_{t u}-\alpha h_{u}, \\
\lambda g_{u}=2 f h_{x u}+g_{u} h_{u} ;
\end{gathered}
$$

thus, (15) is reduced as

$$
h_{t t}-\alpha h_{t}-f h_{x x}+g_{u} h_{x}=0 .
$$

It follows from (17) and (18) that

$$
\lambda f_{u}=0 .
$$

Equation (22) splits into the following two cases.

Case $1(\lambda=0)$. In this case, from (16) we get that $h_{u}=0$; thus, (16)-(20) are all satisfied. Noting that $h$ does not depend on the function $u, f$ and $g$ are two functions of $u$, so from (21) we obtain that

$$
h_{t t}-\alpha h_{t}=0, \quad-f h_{x x}+g_{u} h_{x}=0
$$


TABLe 1: Nonlinear self-adjointness of (3).

\begin{tabular}{lccc}
\hline$f$ & $g$ & $v$ & $\begin{array}{c}\text { Self- } \\
\text { adjointness }\end{array}$ \\
\hline$\forall\left(f_{u} \neq 0\right)$ & $\forall$ & $C_{1}+C_{2} e^{\alpha t}$ & Nonlinear \\
$\forall\left(f_{u} \neq 0\right)$ & $C_{5}$ & $\left(C_{1}+C_{2} e^{\alpha t}\right)\left(C_{3}+C_{4} x\right)$ & Nonlinear \\
$\forall\left(f_{u} \neq 0\right)$ & $C_{5} \int f(u) d u$ & $C_{1}+C_{2} e^{\alpha t}$ & Nonlinear \\
& & $+\left(C_{3}+C_{4} e^{\alpha t}\right) e^{x / C_{5}}$ & \\
$C_{0}(\neq 0)$ & $C_{1}+C_{2} u$ & $C_{1} e^{\left(C_{2} / C_{0}\right) x+\alpha t} u+b(t, x)$ & Weak \\
\hline
\end{tabular}

$\alpha$ is a nonzero constant; $b(t, x)$ satisfies (27).

Equation (23) can be satisfied by taking some appropriate function $h=h(t, x)$. Therefore, we can take $v=h(x, t) \neq 0$ such that $\left.F^{*}\right|_{v=h(t, x, u)}=\lambda F$ holds. Thus, (3) is nonlinear selfadjoint in this case.

Case $2\left(f_{u}=0\right)$. That is, $f$ is a constant with respect to $u$; without loss of generality, we set $f=C_{0} \neq 0$. From (17), we assume that

$$
h(t, x, u)=a(t, x) u+b(t, x) .
$$

Taking into account (16) and (19), we have $a_{t}(t, x)=\alpha a(t, x)$; thus, $a(t, x)=k(x) e^{\alpha t}$. From (20), we deduce that

$$
g_{u}(u)=\frac{C_{0} k^{\prime}(x)}{k(x)},
$$

which implies that $g(u)=C_{2} u+C_{1}$ is a linear function of $u$ and $k(x)=C_{1} e^{\left(C_{2} / C_{0}\right) x}$, where $C_{2}$ and $C_{1}$ are constants. Hence, we have

$$
h(t, x, u)=C_{1} e^{\left(C_{2} / C_{0}\right) x+\alpha t} u+b(t, x) .
$$

Substituting (26) into (22) and using the original equation (3), we derive that $b(t, x)$ satisfies

$$
b_{t t}-\alpha b_{t}-C_{0} b_{x x}+C_{2} b_{x}=0
$$

which is easy to be solved.

In this case, $f(u)=C_{0}$ is a constant, $g(u)=C_{2} u+C_{1}$, and $\lambda=h_{u}=C_{1} e^{\left(C_{2} / C_{0}\right) x+\alpha t}$; therefore, (3) is a weak self-adjoint.

Here, we omit the tedious calculations to obtain the solutions of (23) and (27). In Table 1, we summarize the classification of nonlinear self-adjointness of (3) with the conditions that $f(u)$ and $g(u)$ should satisfy. In what follows, the symbol for all means that the corresponding function has no restrictions and $c_{i}(1 \leq i \leq 5)$ are arbitrary constants.

Thus, we have demonstrated the following statement.

Theorem 3. Let $\alpha$ be a nonzero constant, $f(u)$ an arbitrary function of $u$, and the adjoint equation of (3) given by (14); then (3) is nonlinear self-adjointness with the substitution $v$ and function $g(u)$ given in Table 1.

\section{Symmetries and Conservation Laws}

In this section, we will apply Theorem 2 to construct some conservation laws for (3). First, we show the Lie classical
TABLE 2: Symmetries of (3) for some special choices of $f$ and $g$.

\begin{tabular}{lll}
\hline$f$ & $g$ & Symmetries \\
\hline$u^{-2}$ & $\ln u$ & $X_{3}=x \frac{\partial}{\partial x}-u \frac{\partial}{\partial u}$ \\
$u^{n}(n \neq 0)$ & 1 & $X_{3}=x \frac{\partial}{\partial x}+\frac{2 u}{n} \frac{\partial}{\partial u}$ \\
1 & $X_{3}=u \frac{\partial}{\partial u}$, \\
1 & $X_{4}=t \frac{\partial}{\partial x}+x \frac{\partial}{\partial t}-\frac{1}{2}(t+\alpha x) u \frac{\partial}{\partial u}$ \\
& 1 & $X_{3}=u \frac{\partial}{\partial u}, X_{4}=t \frac{\partial}{\partial x}+x \frac{\partial}{\partial t}-\frac{1}{2} \alpha x u \frac{\partial}{\partial u}$
\end{tabular}

symmetries for (3) for some special choice of $f$ and $g$. Then applying formula in Theorem 2 to the formal Lagrangian (12), and to the symmetries $X_{i}$ and eliminating $v$ by the substitution $v$ given in Table 1 , we obtain the conservation law

$$
D_{t}\left(C^{t}\right)+D_{x}\left(C^{x}\right)=0 .
$$

3.1. Lie Symmetries. Now, let us show the Lie classical symmetries for (3). Based on Lie group theory [22], we assume that a Lie point symmetry of (3) is a vector field

$$
X=\xi(t, x, u) \frac{\partial}{\partial x}+\phi(t, x, u) \frac{\partial}{\partial t}+\eta(t, x, u) \frac{\partial}{\partial u}
$$

on $\mathbb{R}^{+} \times \mathbb{R} \times \mathbb{R}$ such that $X^{(2)} F=0$ when $F=0$, and taking into account (3), the operator $X^{(2)}$ is given as follows:

$$
\begin{aligned}
X^{(2)}= & \xi \frac{\partial}{\partial x}+\phi \frac{\partial}{\partial t}+\eta \frac{\partial}{\partial u}+\eta_{x}^{(1)} \frac{\partial}{\partial u_{x}} \\
& +\eta_{t}^{(1)} \frac{\partial}{\partial u_{t}}+\eta_{x x}^{(2)} \frac{\partial}{\partial u_{x x}}+\eta_{t t}^{(2)} \frac{\partial}{\partial u_{t t}},
\end{aligned}
$$

where

$$
\begin{gathered}
\eta_{x}^{(1)}=D_{x} \eta-\left(D_{x} \xi\right) u_{x}-\left(D_{x} \phi\right) u_{t}, \\
\eta_{t}^{(1)}=D_{t} \eta-\left(D_{t} \xi\right) u_{x}-\left(D_{t} \phi\right) u_{t} \\
\eta_{x x}^{(2)}=D_{x}\left(\eta_{x}^{(1)}\right)-\left(D_{x} \xi\right) u_{x x}-\left(D_{x} \phi\right) u_{x t}, \\
\eta_{t t}^{(2)}=D_{t}\left(\eta_{t}^{(1)}\right)-\left(D_{t} \xi\right) u_{x t}-\left(D_{t} \phi\right) u_{t t} .
\end{gathered}
$$

The condition $X^{(2)} F=0$, when $F=0$, will yield determining equations. Solving these determining equations, we can obtain the symmetries of (3). For $f(u)$ and $g(u)$ arbitrary, the symmetries that are admitted by (3) are

$$
X_{1}=\frac{\partial}{\partial x}, \quad X_{2}=\frac{\partial}{\partial t} .
$$

For some special choices of the functions $f(u)$ and $g(u)$, here we omit the details of routine calculations and present the symmetries (besides $X_{1}$ and $X_{2}$ ) that are admitted by (3) in Table 2. 
TABle 3: Conservation laws of (3) for some special choices of $f$ and $g(n \neq 0)$.

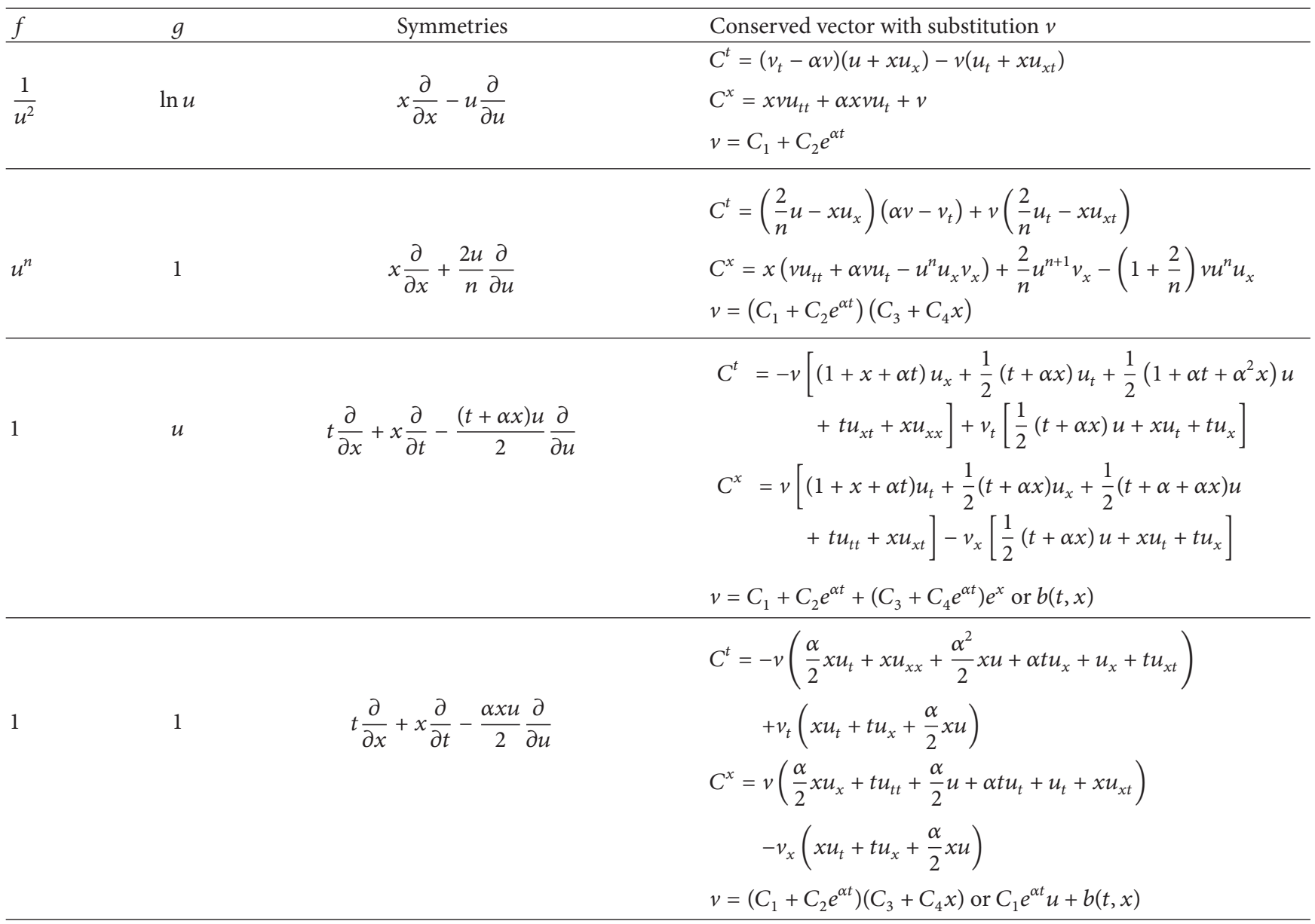

3.2. Conservation Laws. For the symmetries $X$, from the formula (10) in Theorem 2 , we can obtain readily some conservation laws for (3). For example, we take $f(u)=u^{-2}$ and $g(u)=\ln u$; let us construct the conserved vector corresponding to the time translation group with the generator

$$
X_{2}=\frac{\partial}{\partial t}
$$

For this operator, we have $\xi^{t}=1, \xi^{x}=0, \eta=0$, and $W=-u_{t}$. In this case, (3) is nonlinear self-adjoint and becomes

$$
F=u_{t t}+\alpha u_{t}+2 u^{-3} u_{x}^{2}-u^{-2} u_{x x}-u^{-1} u_{x}
$$

The formal Lagrangian is

$$
\mathscr{L}=v\left(u_{t t}+\alpha u_{t}+2 u^{-3} u_{x}^{2}-u^{-2} u_{x x}-u^{-1} u_{x}\right)
$$

and the adjoint equation of (34) is

$$
\mathscr{L}^{*}=v_{t t}-\alpha v_{t}-u^{-2} v_{x x}+u^{-1} v_{x}
$$

Therefore, we obtain the following conserved vector:

$$
\begin{aligned}
C^{t}= & \mathscr{L}+W\left(\frac{\partial \mathscr{L}}{\partial u_{t}}-D_{t} \frac{\partial \mathscr{L}}{\partial u_{t t}}\right)+\left(D_{t} W\right) \frac{\partial \mathscr{L}}{\partial u_{t t}} \\
= & v\left(u_{t t}+\alpha u_{t}+2 u^{-3} u_{x}^{2}-u^{-2} u_{x x}-u^{-1} u_{x}\right) \\
& -u_{t}\left(\alpha v-v_{t}\right)-u_{t t} v \\
= & 2 v u_{x}^{2} u^{-3}-v u_{x x} u^{-2}-v u_{x} u^{-1}+u_{t} v_{t}, \\
C^{x}= & W\left(\frac{\partial \mathscr{L}}{\partial u_{x}}-D_{x} \frac{\partial \mathscr{L}}{\partial u_{x x}}\right)+\left(D_{x} W\right) \frac{\partial \mathscr{L}}{\partial u_{x x}} \\
= & u^{-1} u_{t}\left(v-2 u^{-2} v u_{x}-u^{-1} v_{x}\right)+u^{-2} v u_{x t},
\end{aligned}
$$

where $v$ satisfies (36). The reckoning shows that the vector (37) satisfies the conservation equation (28).

In this case, since (3) is nonlinear self-adjoint, from Table 1 we take $v=C_{1}+C_{2} e^{\alpha t}$. So, the conserved vector is simplified as follows:

$$
\begin{gathered}
C^{t}=2 v u_{x}^{2} u^{-3}-v u_{x x} u^{-2}-v u_{x} u^{-1}+u_{t} v_{t} \\
C^{x}=u^{-1} u_{t} v\left(1-2 u^{-2} u_{x}\right)+u^{-2} v u_{x t} .
\end{gathered}
$$


Particularly, if setting $v=1$, we have

$$
C^{t}=-u_{x} u^{-1}-D_{x}\left(u^{-2} u_{x}\right), \quad C^{x}=u^{-1} u_{t}+D_{t}\left(u^{-2} u_{x}\right) .
$$

Thus, the conserved vector can be reduced to the form

$$
C^{t}=-u_{x} u^{-1}, \quad C^{x}=u^{-1} u_{t} .
$$

If setting $v=e^{\alpha t}$, then the conserved vector (37) is simplified as

$$
\begin{gathered}
C^{t}=\alpha e^{\alpha t} u_{t}-D_{x}\left[e^{\alpha t}\left(u^{-2} u_{x}+\ln u\right)\right] \\
C^{x}=-\alpha e^{\alpha t}\left(u^{-2} u_{x}+\ln u\right)+D_{t}\left[e^{\alpha t}\left(u^{-2} u_{x}+\ln u\right)\right]
\end{gathered}
$$

therefore, it can be reduced as

$$
C^{t}=\alpha e^{\alpha t} u_{t}, \quad C^{x}=-\alpha e^{\alpha t}\left(u^{-2} u_{x}+\ln u\right) .
$$

In what follows, we omit the tedious calculations and list only the conservation laws of (3) for some special choices of functions $f(u)$ and $g(u)$ in Table 3 .

In Table 3 , the function $b(t, x)$ satisfies (27), $v$ and the symmetry are taken from Tables 1 and 2, respectively. The reckoning shows that the vector listed in Table 3 satisfies the conservation equation (28) with corresponding substitution $v$. In the same way above, we can simplify the conserved vector using corresponding substitution $v$.

\section{Conclusions}

Recently, the new outstanding concepts of nonlinear selfadjoint equations, containing quasi-self-adjoint and weak self-adjoint equations, which extend the self-adjointness to a more generalized meaning, have been introduced in order to find formal Lagrangians of differential equations without variational structure. Using these concepts and the general theorem on conservation laws that is, developed recently [8], nonlinear self-adjointness and conservation laws for (3) for different classes of $f(u)$ and $g(u)$ have been discussed. These conservation laws may be useful in mathematical analysis as they provide basic conserved quantity for obtaining various estimates for smooth solutions and defining suitable norms for weak solutions. Furthermore, it could make the construction of the bi-Hamiltonian form easier.

\section{Acknowledgments}

The authors would like to express their sincere gratitude to the referees for many valuable comments and suggestions which served to improve the paper. The first author is partly supported by NSFC under Grant no. 11101111. The second author is partly supported by Zhejiang Provincial Natural Science Foundation of China under Grant no. LY12A01003 and subjects research and development foundation of Hangzhou Dianzi University under Grant no. ZX100204004-6.

\section{References}

[1] A. Barone, F. Esposito, C. G. Magee, and A. C. Scott, "Theory and applications of the sine-gordon equation," La Rivista del Nuovo Cimento, vol. 1, no. 2, pp. 227-267, 1971.
[2] W. F. Ames, R. J. Lohner, and E. Adams, "Group properties of $u_{t t}=\left[f(u) u_{x}\right]_{x}$ " International Journal of Non-Linear Mechanics, vol. 16, no. 5-6, pp. 439-447, 1981.

[3] M. Torrisi and A. Valenti, "Group properties and invariant solutions for infinitesimal transformations of a nonlinear wave equation," International Journal of Non-Linear Mechanics, vol. 20, no. 3, pp. 135-144, 1985.

[4] M. Torrisi and A. Valenti, "Group analysis and some solutions of a nonlinear wave equation," Atti del Seminario Matematico e Fisico dell'Università di Modena, vol. 38, no. 2, pp. 445-458, 1990.

[5] N. H. Ibragimov, Lie Group Analysis of Differential EquationsSymmetries, Exact Solutions and Conservation Laws, CRC, Boca Raton, Fla, USA, 1994.

[6] A. Oron and P. Rosenau, "Some symmetries of the nonlinear heat and wave equations," Physics Letters A, vol. 118, no. 4, pp. $172-176,1986$.

[7] J. G. Kingston and C. Sophocleous, "Symmetries and formpreserving transformations of one-dimensional wave equations with dissipation," International Journal of Non-Linear Mechanics, vol. 36, no. 6, pp. 987-997, 2001.

[8] N. H. Ibragimov, "A new conservation theorem," Journal of Mathematical Analysis and Applications, vol. 333, no. 1, pp. 311328, 2007.

[9] M. S. Bruzón, M. L. Gandarias, and N. H. Ibragimov, "Selfadjoint sub-classes of generalized thin film equations," Journal of Mathematical Analysis and Applications, vol. 357, no. 1, pp. 307-313, 2009.

[10] I. L. Freire, "Conservation laws for self-adjoint first-order evolution equation," Journal of Nonlinear Mathematical Physics, vol. 18, no. 2, pp. 279-290, 2011.

[11] I. L. Freire, "Self-adjoint sub-classes of third and fourth-order evolution equations," Applied Mathematics and Computation, vol. 217, no. 22, pp. 9467-9473, 2011.

[12] N. H. Ibragimov, "Quasi-self-adjoint differential equations," Archives of ALGA, vol. 4, pp. 55-60, 2007.

[13] N. H. Ibragimov, M. Torrisi, and R. Tracinà, "Quasi self-adjoint nonlinear wave equations," Journal of Physics A, vol. 43, no. 44, Article ID 442001, 2010.

[14] N. H. Ibragimov, M. Torrisi, and R. Tracinà, "Self-adjointness and conservation laws of a generalized Burgers equation," Journal of Physics A, vol. 44, no. 14, Article ID 145201, 2011.

[15] M. Torrisi and R. Tracinà, "Quasi self-adjointness of a class of third order nonlinear dispersive equations," Nonlinear Analysis, vol. 14, no. 3, pp. 1496-1502, 2013.

[16] M. L. Gandarias, "Weak self-adjoint differential equations," Journal of Physics A, vol. 44, no. 26, Article ID 262001, 2011.

[17] M. L. Gandarias, M. Redondo, and M. S. Bruzón, "Some weak self-adjoint Hamilton-Jacobi-Bellman equations arising in financial mathematics," Nonlinear Analysis, vol. 13, no. 1, pp. 340$347,2012$.

[18] N. H. Ibragimov, "Nonlinear self-adjointness and conservation laws," Journal of Physics A, vol. 44, Article ID 432002, 2011.

[19] N. H. Ibragimov, "Nonlinear self-adjointness in constructing conservation laws," Archives of ALGA, vol. 7/8, pp. 1-90, 2011.

[20] I. L. Freire and J. C. S. Sampaio, "Nonlinear self-adjointness of a generalized fifth-order KdV equation," Journal of Physics A, vol. 45, no. 3, Article ID 032001, 2012.

[21] I. L. Freire, "New classes of nonlinearly self-adjoint evolution equations of third- and fifth-order," Communications in Nonlinear Science and Numerical Simulation, vol. 18, no. 3, pp. 493-499, 2013. 
[22] P. J. Olver, Applications of Lie Groups to Differential Equations, Springer, New York, NY, USA, 1986. 


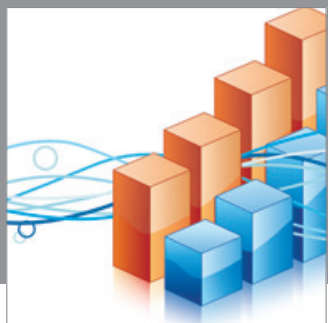

Advances in

Operations Research

mansans

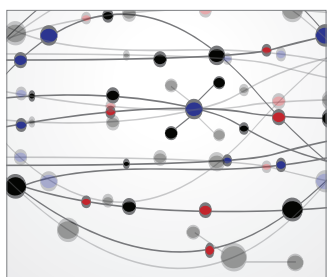

The Scientific World Journal
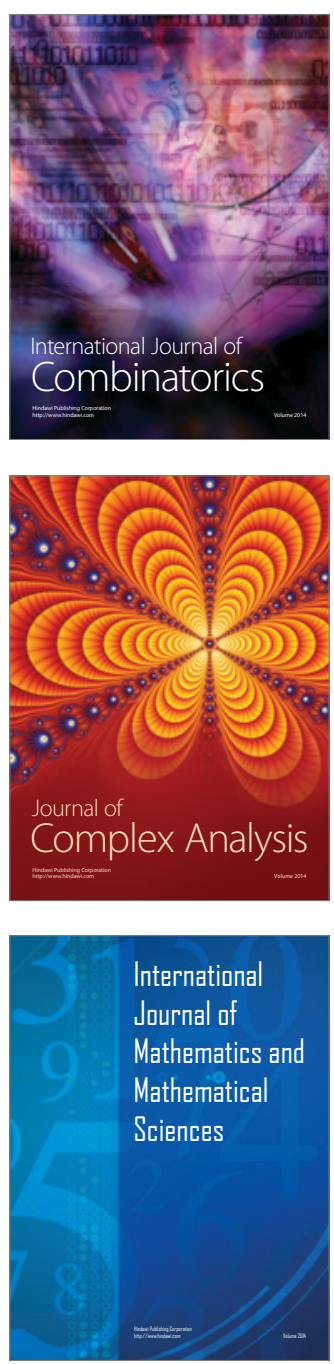
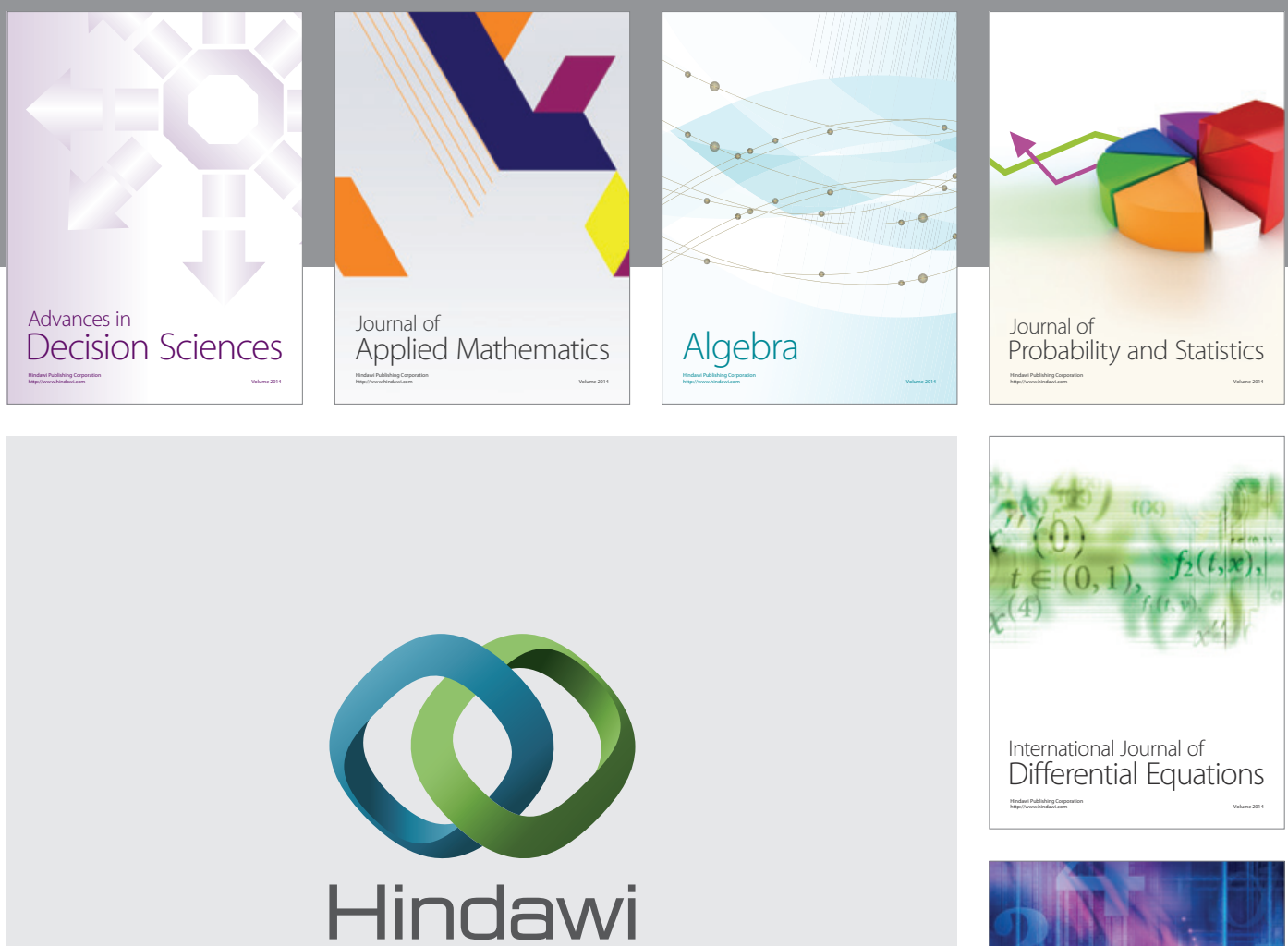

Submit your manuscripts at http://www.hindawi.com
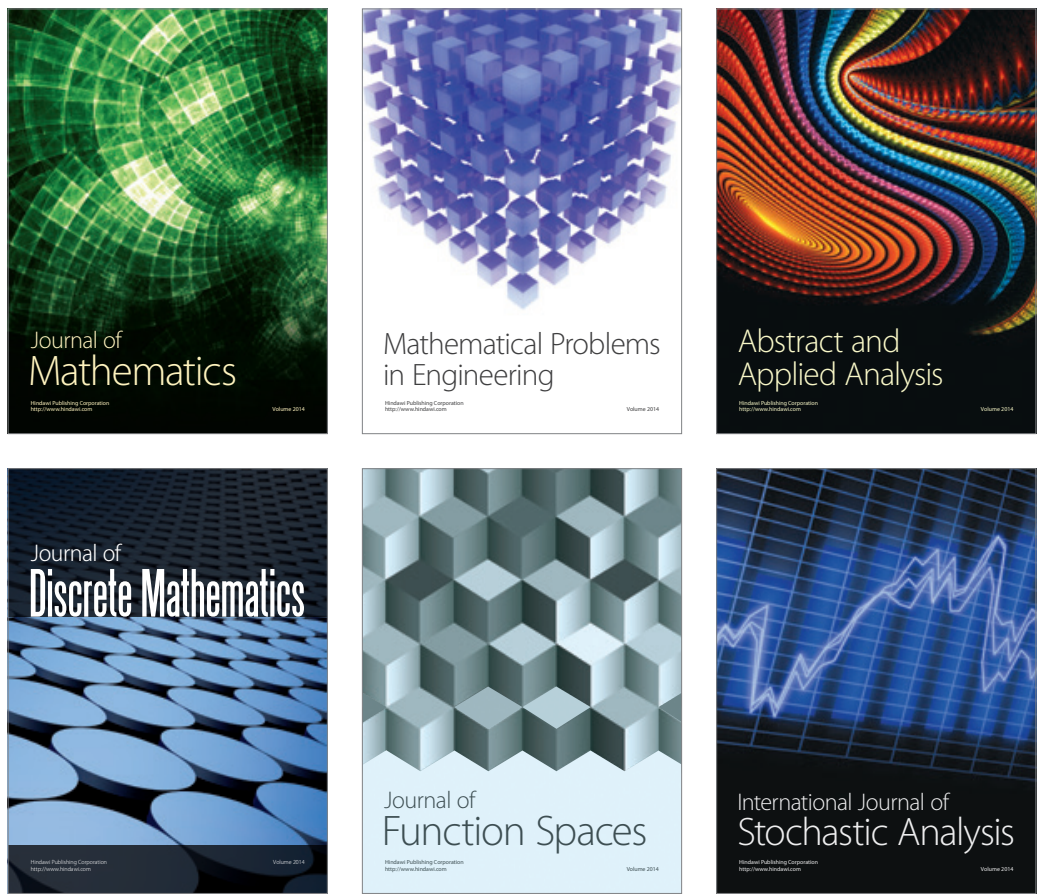

Journal of

Function Spaces

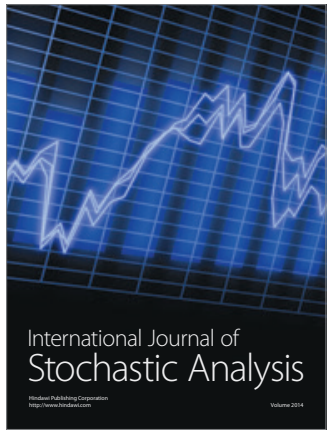

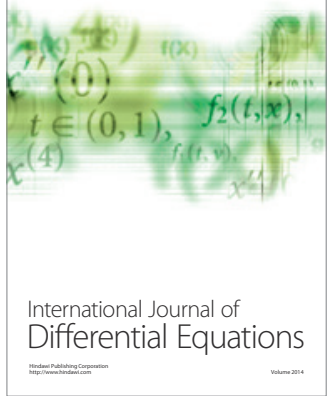
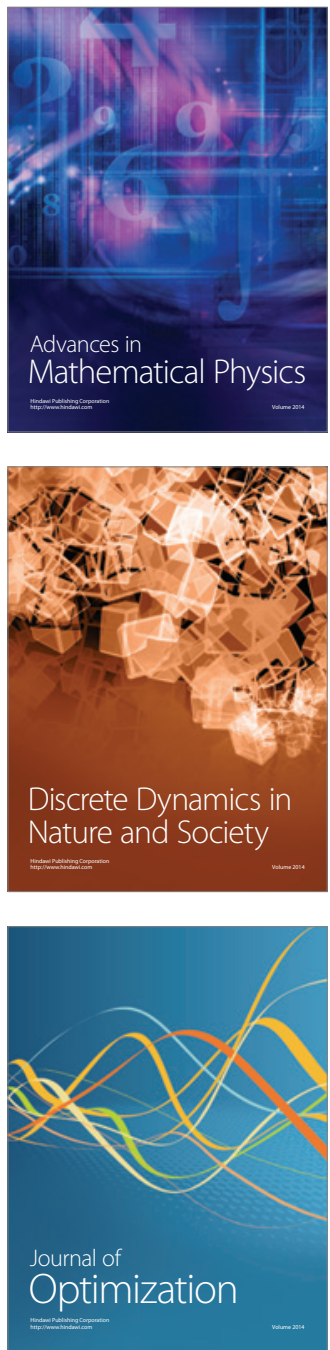